\title{
Georg von Neumayers geophysikalisches Projekt in Australien und Alexander von Humboldt
}

\author{
Von Karl Heinrich Wiederkehr und Wilfried Schröder
}

\section{ZuSAMmenfassung}

Der deutsche Geophysiker und Meeresforscher Georg v. Neumayer (1826-1909) war Zeit seines Lebens von der Antarktis und den angrenzenden Meeren fasziniert. Als mittelloser junger Mann lie $\beta$ er sich als Matrose resp. Steuermann anheuern, um in die Meere der südlichen Hemisphäre gelangen zu können. 1854 legte er dem hochbetagten Alexander von Humboldt (1769-1859) seinen Plan vor, in Melbourne (Australien) eine «Centralstation für Meteorologie, Navigation und Terrestrischen Magnetismus» zu gründen. Neumayer machte dafür wissenschaftiche, praktische und patriotische Gründe geltend. Daß er bei dem großen Forscher und Förderer junger Talente die erhoffte Unterstützung nicht fand, war eine große Enttäuschung für ihn; er fand indessen doch den Weg, seinen Plan zu verwirklichen (1857). - Die Autoren beleuchten diese Episode aufgrund bisher unpublizierter Autographen aus dem Deutschen Museum in München sowie der A.-v.-Humboldt-Forschungsstelle und der Deutschen Staatsbibliothek in Berlin.

In der Humboldt-Forschung mit ihrer umfangreichen Literatur werden viele Fäden der persönlichen Beziehungen des genialen Forschers zu namhaften Wissenschaftlern der verschiedensten Gebiete verfolgt und ein kunstvoll geknüpftes Netz wissenschaftlicher Kommunikation bloßgelegt. Doch eigenartigerweise wurde bisher der kurzfristige Kontakt Georg von Neumayers (1826-1909) zu Alexander von Humboldt in den Jahren 1854 und 1855 in der einschlägigen Literatur nicht erwähnt. Das Zusammentreffen der beiden Wissenschaftler scheint den Humboldt-Biographen bis heute kaum bekannt oder nur von geringer Bedeutung zu sein. Ein neues Licht auf die zu Beginn erfolgversprechende, zuletzt aber glücklose Kommunikation mit A.v. Humboldt werfen einige Schriftstücke von Neumayers Hand, die im Deutschen Museum in München aufbewahrt sind ${ }^{1}$. Der folgende Beitrag will diese offenbar bisher unbekannt gebliebenen Dokumente aufzeigen und historisch auswerten und damit zu neuen Erkenntnissen verhelfen über die 
Einstellung Humboldts zu den maritimen Wissenschaften einerseits und andrerseits über die Zielsetzungen und das Lebenswerk des bayrischen Rheinpfälzers, der 1875 der erste Leiter der Deutschen Seewarte in Hamburg werden sollte und im Jahre 1900 von der bayrischen Krone geadelt wurde. Wohl existieren einige Lebensbilder über G.v. Neumayer; sie sind aber erst ein Stück Biographie und geben keine erschöpfende Auskunft über die Bedeutung dieses großen Mannes.

Auf das Deckblatt des Konvoluts schrieb Neumayer: «Dokumente, die sich auf mein Unternehmen im Jahre 1865 beziehen: Eine Centralstation für Meteorologie, Navigation und Terrestrischen Magnetismus in Melbourne zu gründen.» - Zeitlebens schilderte G.v. Neumayer seine Begegnung mit A.v. Humboldt immer nur mit wenigen Sätzen, und auch seine Biographen beschränkten sich darauf zu berichten, wie Neumayer seine Pläne vor Humboldt entwickelte und dieser dann zustimmte ${ }^{2}$. Nur P. Heidke bemerkte, daß Neumayer eine finanzielle Unterstützung in Berlin versagt blieb $^{3}$. Was an konkreter Hilfe sich Neumayer von Humboldt erhoffte und wie vielschichtiger ihre Kommunikation war, können wir erst diesen Schriftstücken entnehmen. Über den Grund von Neumayers Schweigen werden wir uns noch Gedanken machen müssen.

Das erste Schriftstück aus dem Neumayerschen Konvolut ist der Entwurf eines Briefes an A.v. Humboldt. Die Schrift ist nicht leicht zu entziffern, manchmal sind ganze Silben verkürzt.

Es lautet:

Sr. Hochwohlgeboren dem

Berlin den $10^{\text {ten }}$ Juni 1854

Herrn Baron Alexander v. Humboldt!

Durch den beiliegenden Brief des Herrn Director Rümker, meines hochverehrten Lehrers, werden Sie über mich und meine bisherige Strebsamkeit einige Nachricht erhalten und ich wage es Ihre kostbare Zeit noch weiter zu beanspruchen - um noch Einiges über meine Vergangenheit anzufügen - bevor ich Sie um das Glück bitte, mich Ihnen vorstellen zu dürfen. Von der Überzeugung ausgehend, daß nur Ihr herrlicher Geist es verstanden den Standpunkt der Wissenschaft in unsrer Zeit festzustellen, daß nur der von Ihnen eingeschlagene Weg den Standpunkt erhöhen und wahrhaft große Resultate erzielen kann - beschloß ich nachdem ich die wissenschaftlichen Anstalten meines engeren Vaterlandes Bayern durchlaufen hatte und selbst schon die Resultate meiner Studien über physikalische Gegenstände in München vorgetragen hatte - meine ganze Kraft Ihren erhabenen Ideen über Erweiterung des wissenschaftlichen Feldes zu widmen. Von den nöthigen Mitteln hierzu entblößt mußte ich darauf denken, mir die ferneren Welttheile auf andere Weise zugänglich zu machen und in meinem $23^{\text {ten }}$ Jahre wurde ich Matrose - lange Reisen 
im atlantischen Ocean gewöhnten mich an dieses rauhe Leben und vor drei Jahren faßte ich den Entschluß - das Australische Festland zum Gegenstand meiner Beobachtungen zu machen - ein Entschluß, der um so schwieriger zu realisieren war, da die mittlerweile aufgefundenen Goldfelder jedes geringe Kapital verschwinden ließen gegenüber den bedeutenden Ausgaben, welche das Reisen verursachte. Darauf hingewiesen mit den Händen zu erarbeiten, was ich auf meine Zwecke wieder verwenden wollte, machte ich als Matrose und später als practischer Goldgräber wenigstens so viel - um einige Fußreisen in den Küstenstrichen des Australischen Continents ausführen zu können. Ohne Instrumente und Hilfsmittel zum Transport sind meine Beobachtungen unbedeutend, meine Sammlungen arm geblieben - allein ich hoffe doch wagen zu dürfen Euer Hochwohlgeboren um einen Augenblick der Unterhaltung zu bitten, den Muth dazu giebt mir die Hoffnung, doch vielleicht etwas unter meinen gesammelten Materialien zu haben, was in Ihrer segensreichen wissenschaftlichen Thätigkeit Bedeutung gewinnen könnte.

Allein noch ein Weiteres veranlaßt mich zu diesen Zeilen - gerne möchte ich für meine nächsten wissenschaftlichen Unternehmungen Ihren unschätzbaren Rath zur Sache haben um mich durch dieß Glück gestärkt von Neuem auf dem Australischen Continent der Wissenschaft und Ihren Forschungen zur Verfügung zu stellen.

Gewiß werden Sie dem strebenden Schüler die Stärkung des Vertrauens auf seine Erfolge - die in dem Rath des vollendeten Meisters liegt - nicht versagen.

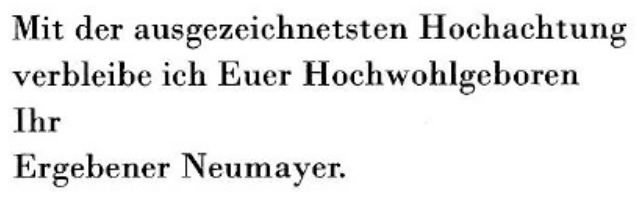

Der Brief wirkt in Stil und Ausdruck etwas hölzern und befangen; doch nimmt dies nicht wunder, wenn man bedenkt, daß hier ein junger, noch ganz unbekannter Wissenschaftler dem gekrönten Haupt der Wissenschaften, die sich mit der Erde und ihren Phänomenen befaßten, zum erstenmal gegenübertritt. Ende Januar 1854 war Neumayer von seiner Australienreise zurückgekehrt, fest entschlossen, in Melbourne, der Hauptstadt der aufblühenden englischen Kolonie Victoria, eine geophysikalische und nautische Forschungsstation zu errichten. Die Erlebnisse bei jenem ersten Besuch des entlegenen Erdteils, besonders die abenteuerlichen Wochen in den Goldfeldern von Bendigo-Diggins schilderte er später breit und anschaulich in dem Zeitschriftenartikel «Ein deutscher Seemann und die Erschließung der Goldausbeute in Autralien» (1907) ${ }^{4}$. In jenen Jahrzehnten suchte fast jeder Forschungs- und Entdeckungsreisende Rat bei A.v. Humboldt und hoffte auf Anteilnahme und Unterstützung. Anscheinend hatte Georg Neumayer ein paar Dinge - gewissermaßen als Aufhänger für ein persönliches Gespräch mit A.v. Humboldt, vielleicht sogar als Präsent - aus seinen Sammlungsstücken ausgewählt. Welcher Art diese waren, erfahren wir nicht. 
Zum besseren Verständnis wollen wir jedoch zuerst Neumayers Lebensweg um einige Jahre zurückverfolgen. Sein Lehrer Johann von Lamont (1805-1879) hatte in ihm das besondere Interesse für erdmagnetische Forschungen geweckt. Die Gaußsche Theorie konnte Neumayers Ansicht nach nur durch umfangreicheres Beobachtungsmaterial in der Nähe des südlichen Magnetpols verfeinert werden. Auf den Studenten in München übte auch der Wirtschaftswissenschaftler Friedrich List (1789-1846), der sich für die Öffnung des damals im Einigungsprozeß befindlichen Deutschlands zur See hin einsetzte, einen starken Einfluß aus. Schon früh reifte in Neumayer der Plan, der deutschen Seefahrt und den maritimen Wissenschaften den Anschluß an das Niveau der führenden Seemächte zu ermöglichen. Konsequent nahm er das harte Los auf sich, als Seemann von der Pike auf zu dienen. Denn nur in der Synthese von Praxis und Theorie war in seinen Augen dieses Ziel erreichbar. In Hamburg machte Neumayer innerhalb von zwei Monaten das Steuermannsexamen bei dem bekannten Astronomen und Leiter der Navigationsschule Charles Rümker (1806-1862) und wirkte sogar als Hilfslehrer, um seinen ihm freundschaftlich verbundenen Lehrer zu entlasten ${ }^{5}$. Dafür, daß Neumayer gerade Australien und Melbourne als zukünftige erfolgversprechende Wirkungsstätte wählte, gibt es neben dem schon erwähnten geomagnetischen Aspekt noch andere Gründe. Charles Rümker war in der englischen Kolonie Neusüdwales (Australien) an der Privatsternwarte des Gouverneurs früher tätig gewesen und konnte so manch guten Ratschlag geben. Die Auswandererwelle nach Australien hatte Mitte der fünfziger Jahre in Deutschland einen Höhepunkt erreicht, und von dort kam die Kunde über gute Startmöglichkeiten und manches gemachte Glück $^{6}$. Das Auswanderergeschäft lag großenteils in der Hand des Hamburger Handelshauses Johann Cesar Godeffroy und Sohn, seine Schiffe segelten nach Australien und in die Südsee, und die Inhaber waren sehr daran interessiert, daß G. Neumayer die Kapitäne auf ihren Routen nautisch beriet ${ }^{\text {? }}$.

Bei dem obigen Brief erscheint uns noch wichtig festzuhalten, daß Neumayer hier schon erkennen läßt, daß er kein eigenes Kapital für sein geplantes Unternehmen besitze und daß ihm eine Ausrüstung mit leistungsfähigen Instrumenten Vorbedingung für ein Gelingen sei. Das Antwortschreiben Humboldts, das der Bitte Neumayers um eine persönliche Begegnung entsprach, ist uns erhalten geblieben und im Besitze der La Trobe Collection State Library of Victoria. Wir geben es hier wieder ${ }^{8}$. 


\begin{abstract}
Sie werden mir eine grosse Freude machen, wenn Sie mich übermorgen Mittwochs um 1 Uhr mit Ihrem Besuche beehren wollen. Die Freude besteht nicht bloss darin, Ihnen für einen freundschaftlichen Brief des vortrefflichen Dir. Rümcker danken zu können. Sie gründet sich hauptsächlich auf der Achtung, die Ihrem Muthe, Ihrer schönen Energie des Characters gebührt, mit der Sie sich Entbehrungen auferlegt geistige Bedürfnisse zu befriedigen. Solche Bestrebungen erregen ein rein menschliches Interesse

Montag eben

nach Sanssouci zurückgekehrt.

Ich werde Sie nicht in Potsdam,

Ihr gehorsamster

sondern bei einem vielbewegten

A.v. Humboldt

Leben in Berlin empfangen
\end{abstract}

Wie Georg Neumayer uns in seinem Buche «Auf zum Südpol» mitteilt, wurde er von Humboldt Mitte Juni in der Oranienburger Straße 67 empfangen ${ }^{9}$. Die besondere Atmosphäre des Zimmers schilderte uns Wilhelm Foerster (1832-1921), als er im Herbst 1855 als junger Assistent der Berliner Sternwarte dort weilte. Über dem Sofa hing eine kleine reizende Skizze des väterlichen Hauses in Tegel, und in der gegenüberliegenden Ecke stand die Büste des verehrten Freundes François Arago ${ }^{10}$. Beim Vortragen seiner Pläne wies unser tatendurstiger Wissenschaftler und Seemann sicherlich darauf hin, daß er mit seinen Untersuchungen zum terrestrischen Magnetismus eigentlich die Arbeit A.v. Humboldts fortsetzen wollte, die er auf seiner Rußlandreise (1829-1830) begann und die dann im Göttinger Magnetischen Verein mit Karl Friedrich Gauß und Wilhelm Weber zu einer weltumspannenden Organisation und Kooperation gedieh und schließlich dank der Initiative eines Edward Sabine und des Wagemutes von James Clark Ross mit der Antarktisexpedition (1839-1843) so ruhmvoll endete ${ }^{11}$. Hinsichtlich seiner geplanten meteorologischen Arbeiten fand Neumayer wohl ein nicht minderes Interesse vor. Humboldts Studien über atmosphärische Physik und barometrische Oszillationen hatten das Verständnis für die Passatwinde erschlossen und den grundsätzlichen Unterschied zwischen Küsten- und Binnenklima formuliert. Auf sein Betreiben hin kam das meteorologische Institut in Berlin zustande, eine Art Zentralstation für Preußen, dessen Leitung bald Heinrich Wilhelm Dove (1803-1879) übernahm. Der gelernte Physiker hatte sich Meteorologie und Klimatologie als Spezialgebiete gewählt und wurde mit seinen grundlegenden Arbeiten (die 
später allerdings einer Korrektur bedurften), insbesondere mit seinem Drehungsgesetz der Winde, zu einer international anerkannten Autorität ${ }^{12}$.

Bei seinem dritten Punkt, der neben Forschungen auch die praktischen Belange der Seefahrt mit einbezog - nämlich eine Beratungsstelle für meteorologisch-geophysikalische Navigation in Melbourne einzurichten -, fand Neumayer vermutlich in Humboldt ebenfalls einen aufmerksamen Zuhörer und Gesprächspartner. Durch Ch. Rümker war A.v. Humboldt schon 1849 mit den Arbeiten und Absichten Matthew Fontaine Maurys (1806-1873) bekannt geworden: durch systematische empirische Untersuchungen und Auswertungen die günstigsten Routen für die Segelschiffe auf den Weltmeeren zu finden und die Reisezeiten zu verkürzen. Maury gelang es auf der Brüsseler Konferenz 1853, die führenden Seemächte für sein Vorhaben zu gewinnen. Auch Preußen trat später den Brüsseler Vereinbarungen bei. In seinen «Wind and Current Charts», die 1847 zum erstenmal und dann später in mehreren erweiterten Auflagen erschienen, und in seinem zusammenfassenden Werk «The Physical Geography of the Sea and its Meteorology» (1856) berief sich Maury direkt auf Alexander von Humboldt, den Begründer der Physischen Geographie ${ }^{13}$. Zu seiner Legitimation zitiert er einen Brief Humboldts an den nordamerikanischen Generalkonsul in Leipzig Dr. Flügel ${ }^{14}$ :

\footnotetext{
«I beg you to express to Lieut. Maury, the author of the beautiful Charts of the Winds and Currents, prepared with so much care and profound learning, my hearty gratitude and esteem. It is a great undertaking, equally important to the practical navigator and for the advance of meteorology in general. It has been viewed in this light in Germany by all persons who have a taste for physical geography.»
}

Welche Bedeutung A.v. Humboldt den Luftbewegungen über dem Meer, der Flora und Fauna und den ozeanischen Strömungen beimaß, wird uns in seinem Alterswerk «Kosmos» eindrucksvoll vor Augen geführt ${ }^{15}$. Das Meer fasziniert ihn, es ist ihm der «Anblick des Grenzenlosen und Unermeßlichen, welchen jede Seefahrt darbietet. Wer zu geistiger Selbstthätigkeit erweckt, sich gern eine eigene Welt im Innern bauet, den erfüllt der Schauplatz des freien, offenen Meeres mit dem erhabenen Bilde des Unendlichen.» ${ }^{16}$

Wir gehen wohl nicht fehl, wenn wir annehmen, daß A.v. Humboldt bei der Unterredung im Juni 1854 G. Neumayers Plänen positiv gegenüberstand und dieser sich ermuntert fühlte, eine umfassendere Denkschrift auszuarbeiten. Am 6. Dezember 1854 schickte er diese nach Berlin. Die erste persönliche Begegnung mit dem Altmeister der Geowissenschaften stimmte 
Neumayer zunächst recht optimistisch ${ }^{17}$. Doch die weitere Entwicklung, gewissermaßen die zweite Phase, verlief anders als erwartet. Lassen wir Georg Neumayer den Fortgang der Dinge selber schildern.

Zwei Briefentwürfe aus dem Konvolut im Deutschen Museum München der erste an Professor Ehrenberg, der zweite an A.v. Humboldt - verdeutlichen die Lage Neumayers und seine Gemütsverfassung.

An Seine Hochwohlgeboren dem Herrn

Professor Dr. von [?] Ehrenberg!

Als ich vor 12 Wochen an Seine Excellenz den Herrn Baron Alexander von Humboldt eine kurze Entwicklung meiner Absichten und Pläne einsandte wagte ich es auch an Sie einige Zeilen zu richten, um mir Ihre viel vermögende Fürsprache zu erbitten. Ich würde überhaupt nicht den Muth gehabt haben mit meinen Ansichten und Wünschen vor die ausgezeichnetsten Männer unseres Vaterlandes zu treten, wäre nicht in dem Bewußtsein, für eine gewichtige Sache zu streben, ein Sporn gelegen. Euer Hochwohlgeboren gegenüber war es das Vertrauen auf Thre Güte, das mich so unbescheiden sein ließ, Sie mit meinen Angelegenheiten zu beanspruchen. - Jenes Vertrauen stützte sich auf die freundliche Theilnahme, welche Sie bei meinem Besuche in Berlin mir zugewendet haben und auch das innige Verhältniß, in welchem ich zu Ihrem Schwager, Herrn Alexander Rose gestanden habe und noch stehe. Eine ausführliche Besprechung meiner Pläne hielt ich deshalb nicht für nöthig meinem Schreiben an Sie folgen zu laßen, weil ich voraussetzte, daß Eure Hochwohlgeboren durch die innige Beziehung, in welcher Sie zu seiner Excellenz dem Herrn Alexander von Humboldt stehen, von Allem, was meine Ansichten und Wünsche angeht, bei einer etwaigen Besprechung genau unterrichtet werden würden.

Bis heute ist mir keine Rückäußerung zugegangen, die mich aus der unangenehmen Lage, in welche mich die Ungewißheit versetzt, hätte bringen können.

Noch Einmal bin ich um deßwillen so unbescheiden Euer Hochwohlgeboren mit einer Bitte zu belästigen, deren Erfüllung nur in wenigen Zeilen besteht. Gewiß haben Sie die Gewogenheit mir mitzutheilen, wie weit meine Angelegenheiten gediehen sind und ob ich überhaupt nur Etwas zu erwarten habe. - Mag die Entscheidung fallen, wie immer sie wolle, ich werde den Australischen Continent wieder besuchen, von der festen Überzeugung geleitet, dort einen mühsamen aber erfolgreichen Beruf leben zu können. [.?.] werde ich vorher jedenfalls mein Versprechen einlösen und so wohl Euer Hochwohlgeboren als auch Ihrem Herrn Schwager in Schönebach [gemeint ist vermutlich Schöneberg] meine Aufwartung machen, um Aufträge für Herrn Alexander Rose entgegen nehmen zu können.

Entschuldigen Sie meine Dreistigkeit - in dem ich Sie zu wiederholten Malen belästigte, und empfehlen Sie mich Ihrer Werthen Familie auf das freundlichste.

Mit der ausgezeichnetsten Hochachtung Euer Hochwohlgeboren ganz ergebener Neumayer

Frankenthal den $23^{\text {ten }}$ Februar 1855 
An Sr. Excellenz dem Herrn Baron

Alexander von Humboldt.

Kaum kann ich es wagen Eurer Excellenz wieder mit einem Schreiben zu nahen; nur die Liebe für die Sache, der ich mich gewidmet, nur die Überzeugung durch die in meinem Schreiben vom $6^{\text {ten }}$ December entwickelten Pläne zu ersprießlichen Resultaten zu gelangen gibt mir den Muth dazu.

Vor Eurer Excellenz hohen Weisheit glaubte ich mein Herz ausschütten zu können; für meine Überzeugung, zu welcher ich durch die herbsten Erfahrungen gelangt war, glaubte ich in die Schranken treten zu müßen, und wie in dieser Äußerung des Gefühls und des Verstandes eine doppelte Richtung liegt, so war auch ein doppeltes Wagniß damit verknüpft. Sr. [Excellenz] unbekannt und ohne jede Bedeutung wagte ich mit dem vollen innigsten Vertrauen zu einem Manne aufzublicken, deßen Name mich bisher nur mit der höchsten Verehrung erfüllte; ungeübt und in wenig gewandtem Sprechen entwickelte ich meine wissenschaftlichen Ansichten vor dem höchsten Schiedsrichter der Wissenschaft. In solchen Fällen ist es schwer überall die Befangenheit zu verbergen und das richtige Maaß zu halten. Leicht wird die Äußerung des Gefühls untreu, die Darlegung wissenschaftlicher Ansichten schülerhaft. In wie weit es mir gelungen in meinem Schreiben vom $6^{\text {ten }}$ December 1854 diese [.?.] zu werden - wie weit ich dem Wagniße verfallen, vermag ich nicht zu entscheiden - nur soviel bleibt mir gewiß: Eurer Excellenz edles Gemüth werden mein Vertrauen entschuldigen, wenn auch meine Wissenschaft in der Prüfung nicht bestehen sollte. Das herannahende Frühahr mahnt mich meiner Vorsätze eingedenk zu sein und die Rüstung zu meinen Unternehmungen zu betreiben. Von Eurer Excellenz gütiger Entscheidung wird es abhängen, ob ich mit größerem oder geringerem Erfolg zu streben hoffen kann. - Mein Mut bleibt ungebrochen, weil er sich auf die uneigennützigste Liebe zu einer edlen Sache stützt.

Mit der aufrichtigsten, wärmsten Verehrung

Eurer Excellenz

Ergebenster

Neumayer

Frankenthal, den $24^{\text {ten }}$ Februar 1855

Christian Gottfried Ehrenberg (1793-1876) war mit A.v. Humboldt freundschaftlich eng verbunden. Zusammen mit dem Mineralogen Gustav Rose hatte Ehrenberg als Arzt und Zoologe Humboldt auf der russisch-sibirischen Reise begleitet. Als Entdeckungsreisender in Ägypten und als Erforscher der Infusorien war er schon früh weltweit bekannt geworden, als Mikrobiologe wies er den Naturwissenschaften neue Wege. Bei der Berliner Akademie 
bekleidete Ehrenberg das Amt eines der beiden ständigen Sekretäre, und die Universität machte ihn 1855 zu ihrem Rektor ${ }^{18}$. Ehrenbergs Gattin Julie war eine geborene Rose und stammte aus Wismar. Über ihren Bruder Alexander ist uns nichts weiteres bekannt.

Der zweite Brief Neumayers ist in seinem offenen Aussprechen der innersten Antriebe und Gefühlsregungen etwas ungewöhnlich. Bei der Kommentierung des Neumayerschen Briefes an Hans Ferdinand Maßmann werden wir auf diesen Punkt noch einmal zurückkommen. Die Denkschrift, die G. Neumayer an A.v. Humboldt abschickte, ist bis heute noch nicht aufgefunden worden, ebenso wie die spätere an den bayrischen König Maximilian II. gerichtete. Die dritte, zuletzt verfaßte «kürzere» Denkschrift, datiert mit dem 26. August 1856 und dem Hamburger Senator Gustav Godeffroy überreicht, ist uns erhalten geblieben und liegt in den Akten des Hamburger Staatsarchivs ${ }^{19}$.

Der Brief an Maßmann ist das längste Schriftstück im Münchener Konvolut. Es muß gesehen werden, daß M. am 27.2.1855 eine Kopie des Humboldtschen Briefes (26.2.) an Neumayer gesandt hat. N's Hinweis «den Wortlaut des Briefes von St. Excellenz» (s.S.102) bedeutet, daß er die Maßmannsche Abschrift des Humboldtbriefes vom 26.2. an M. zurückschickte.

Über den Verbleib des Humboldtschen Briefes selbst ist bis heute nichts bekannt. Auch wenn uns die Entzifferung des Neumayerschen Briefentwurfes an einigen Stellen nicht ganz gelungen ist, halten wir ihn als wichtige Primärquelle für wert genug, vollständig wiedergegeben zu werden.

Spielt für A.v. Humboldt die Kommunikation mit G. Neumayer auch nur eine untergeordnete Rolle in seinen wissenschaftlichen Beziehungen, kann ihre Betrachtung vielleicht doch als Mosaiksteinchen nützlich sein.

Entwurf des Schreibens an H.F.Maßmann:

Hochgeehrter Herr Professor!

Frankenthal den $2^{\text {ten }}$ März 1855

Heute erhielt ich Ihren Werthen Brief vom 27ten Februar und [.?.] keinen Augenblick denselben zu beantworten, um Sie so schnell als möglich von Allem zu benachrichtigen, was meine Angelegenheiten anbelangt. 
Das Einzige was ich seit Absendung meines Briefes an Se. Excellenz dem Herrn von Humboldt zur Antwort erhielt, waren Ihre werthen Zeilen vom 17. December 1854, worin es gleich im Anfange hieß: Ich will nicht länger zögern Ihnen anzuzeigen, daß ich Ihre mir anvertraute Eingabe an den Geh. Rath Freiherrn Alexander von Humboldt am 10. d. M. [.?.] überreicht habe. Nach der raschen und liebenswürdigen Weise des nun 86jährigen Greises werden Sie entweder schon Antwort in Händen haben oder dieselbe zweifelsohne demnächst erhalten. Möge dieselbe Ihren Wünschen vollkommen entsprechen. Niemand wünscht dieß mehr als ich, der ich durch meine Söhne innerlich daran den aufrichtigsten wärmsten Antheil nehme. - Als wir uns im Sept. etc. etc.

Durch diese Mittheilung vollkommen beruhigt ließ ich 6 Wochen vorübergehen ohne weitere Schritte in meiner Sache zu thun. - Als ich aber weder von Sr. Excellenz, noch von Herrn Prof. Ehrenberg und von Dove nach Ablauf dieser Zeit eine Nachricht erhielt, wendete ich mich an Sie, um nur Einiges über das Geschick meiner Eingabe zu erfahren und in einem weiteren Schreiben, dessen Datum ich nicht genau mehr weiß, bat ich Sie um eine gefällige Mittheilung. Meine Besorgniße und meine Unruhe stiegen von Tag zu Tag. Hundert Fragen warfen sich auf, die alle zu keinem Resultate führen konnten. Der Umstand, daß ich von Ihnen keine Nachricht bekam, schien mir zu beweisen, daß meine Sachen schlecht stünden, jedoch hatte ich darüber keine Gewißheit. Das herannahende Frühjahr, das mich zur neuen Thätigkeit mahnte, machte eine Gewißheit über die Sachlage wünschenswerth und ich faßte deshalb den Entschluß sowohl an Se. Excellenz als auch an Herrn Prof. von Ehrenberg Anfragen zu richten. Am 24 ${ }^{\text {ten }}$ Februar 1855, also 12 Wochen nach meiner ersten Eingabe wagte ich die erste direkte Anfrage zu stellen.

Ich gebe Ihnen hier den Wortlaut des Briefes von Sr. Excellenz. Urtheilen Sie, hochgeehrtester Herr Professor, aus diesen Zeilen auf meine Empfindungen. - Seine Excellenz machen mir in dem Brief den Sie so gütig waren zu [.?.], den Vorwurf der Sentimentalität. Nichts ist meinem Wesen fremder als das. Was ich schrieb war meine tiefe Empfindung und sollte mir nur eine Antwort erwirken und mich aus der Ungewißheit reißen. - Auch meine Kopie [.?.] liegt darin nicht; - ich fand es erklärlich, daß der hochbetagte Mann im Interesse der Wissenschaft mit seiner Zeit sparsam ist und mir dem unbekannten, ihm fern stehenden Menschen keine weitere Aufmerksamkeit zuwenden kann. Laßen Sie mich nun noch einiges über die Eingabe anfügen. Mit Absicht ließ ich dieselbe unversiegelt, als ich so frei war, Sie mit der [.?.] zu belästigen. - Ich wollte Sie von Ihnen gelesen und beurtheilt wissen. - Gewiß waren Sie auch so gütig, meiner Entwicklung zu folgen und ich erlaube mir Ihnen nur einzelne Punkte in's Gedächtnis zurück zu rufen.

Was beabsichtigte ich durch diese Eingabe? Es konnte mir nicht einfallen eine genaue Wissenschaftliche Erörterung beifügen zu wollen. Wie hätte ich es wagen sollen dem größten Mann der Wissenschaft gegenüber mich über den Stand einer speciellen Wissenschaft der Navigation auszusprechen um daran anknüpfend die zu verfolgende Richtung streng zu begründen. Das hieße ja doch einen Tropfen Wasser in's Meer zu tragen. Wohl aber habe ich meine Wünsche streng formulirt, nachdem ich vorher in meiner Einleitung die leitenden Motive erörtert hatte. Die Überzeugung, daß durch vergleichende Beobachtungen vorzüglich auf die weitere Entwickelung der Wissenschaft zu wirken sei hatte ich aus den Werken des Herrn von Humboldt geschöpft und Maury's Wege und Erfolge bieten zur Verfolgung dieser Richtung die vorzüglichsten Muster. Eine meteorologische Station in Melbourne errichtet, dem Hafen, in welchem sich Schiffe aller Nationen und der 
vorzüglichesten Bauart treffen, läßt durch Sammeln des aus Schiffsjournalen gezogenen Materials, bedeutende Erfolge erwarten. -

Wie es um die deutsche Navigation steht, wissen Sie durch Ihre Söhne und werden es noch mehr erfahren, wenn dieselben erst einmal von ihrer jüngsten Reise zurückkehren. Wir sind zurück, außerordentlich zurück und es ist vollkommen an der Zeit, wenn wir ernstlich suchen uns hierin mit andern Nationen gleichzustellen, - ein Bestreben, das nur wissenschaftliche Untersuchungen auch dieser Richtung mit Erfolg krönen können. Die neuesten Erfahrungen haben mir wieder gezeigt, daß ich die Sachlage richtig beurtheile. Auf Thre gütige Anzeige verschrieb [.?.] ich mir augenblicklich jenes neue Lehrbuch der Navigation von M.F.Albrecht und Vierow. In der That ein ausgezeichnetes Werk, was theoretische Begründung und logische Ordnung anbelangt - aber was wurde dadurch erreicht. Eine Erweiterung der Navigation gewiß nicht, denn die streng wissenschaftliche Richtung dieses Werkes macht es zur Schule unserer Seeleute in Nautischer Astronomie nicht so tauglich als unseres vortrefflichen Rümkers Handbuch. Für richtige Wahl unserer Wege, für zweckmäßigen Gebrauch der Segelkraft, für Schiffskonstruktion ist darin Nichts geboten. Höher gebildete Officiere finden in den Arbeiten Bohnenbergers, [.?.], Littrows etc. etc. vortreffliche Mittel zur Weiterbildung.

Die Vereinigung der Praxis und Theorie thut in Deutschland wie fast in allen Disciplinen so auch in dieser, vor Allem Noth. Wodurch ist dieß zu erreichen? Nur dadurch daß wir uns wissenschaftlich gebildet die Erfahrungen einer praktischen Bahn aneignen. Deßhalb opferte ich Alles um diesem Zwecke zu leben, deßhalb freute ich mich so herzlich Ihre trefflichen Söhne auf gleichem Wege zu sehen.

Meine Erfahrung hat mich gelehrt, daß wissenschaftliche Männer, emsige Forscher und wackere Köpfe für die Erwerbung der Wissenschaft unserer Nation angehörig nun aber von einer fremden Nation unterstützt - mehr und mehr ihr Vaterland vergaßen und oft gelangen wir erst durch Dritte zu den Resultaten ihrer Forschungen und dieß passiert nicht zum Vortheile unserer Wissenschaft. Von den vielen Beispielen erwähne ich nur Rümker und Müller, welche beide [.?.] erster als Government-Astronom in Sydney, letzter als Government-Botaniker in Melbourne durch eine Reihe von Jahren thätig waren. Rümker war während 7 Jahren aus aller Verbindung mit Deutschland und Müller sendet seine [.?.] Berichte und herrlichen Sammlungen nach London, während in Deutschland nur wenig von ihm bekannt wird. Muß das den Deutschen nicht schmerzen? und gewiß haben Sie, hochgeehrtester Herr Professor, dasselbe patriotische Gefühl, welches mich trieb bei der Ausführung meines Planes eine Verbindung mit der Berliner Academie zu gründen. Folgende Stelle meiner Eingabe enthielt meine Wünsche: «Könnte es mir gelingen an einer in Melbourne zu errichtenden meteorolog.-magnetischen Station eine Thätigkeit zu vertreten, welche die Befriedigung australischer Interessen in sich schließt, könnte ich in innigster wissenschaftlicher Verbindung mit meinem Vaterlande ...»

Wie Sie sehen war von einer Empfehlung an Beaufort, die Gesellschaft, Faraday in meinem Bericht keine Rede, weil diese Männer keinerlei Beziehung zu den Australischen Verhältnißen haben. - Sobald ich eine gütige Rückäußerung über meine Pläne, deren [.?.] Hebel ich entwickelt hatte, und die Erlaubniß erhalten hätte, mich weiter in dieser Sache an Se. Excellenz wenden zu dürfen, würde ich ein [.?.] Schreiben eine kurze Darlegung meiner Wünsche und eine Bewerbung [.?.] in englischer Sprache abgefaßt und Sr. 
Excellenz zur gütigen Begutachtung und [.?.] vorgelegt haben. Gewiß liegt darin nicht der Wunsch «daß eine neue Lage geschaffen wird», sondern lediglich die Bewerbung um bestehende Stellen, welche ich in meiner Eingabe [.?.] aufführte. So liegt auch in der Empfehlung, welche ich mir von Sr. Excellenz erbat, ein Sinn; man würde nicht gezögert haben, einen Mann, der von Sr. Excellenz empfohlen worden ist, in Melbourne vorzuschlagen, wenn [.?.] Alles was ich dadurch erreichen konnte, war eine Überfahrt nach Australien mit Aussicht auf wissenschaftliche Verwendung daselbst. Was ich weiter aus einer somit errungenen Stellung zu erreichen vermag, hängt von meiner Befähigung und meinem Muthe ab.

Der zweite Theil meines Wunsches betraf die Academie der Wissenschaften in Berlin. Die größte Schwierigkeit besteht darin deutlich auszusprechen was ich wünsche, die Motivirung war in der Eingabe ausführlicher als in diesen Zeilen. Nun kommen wir an den Hauptpunkt meiner Angelegenheit: die Möglichkeit der Ausführung und ich mußte dieselbe, so wie sich mein Wunsch in zwei Theile theilt, in zwei getrennten Sätzen beantworten.

Die Errichtung einer solchen Station, wie oben bezeichnet, kann nur Sache der Colonial-Regierung sein und [.? .] vertraut [.?.] mit den Bestrebungen und der politischen Lage der Colonien weiß ich auch, daß man sich in Melbourne und Sydney nur ungern einen Beamten oder Mann von Wissenschaft [.?.] läßt (dieses habe ich in der Eingabe nicht erörtert, weil ich es meinem Zweck nicht dienlich erachtete). Man wählt dort gern selbst und giebt dabei übrigens gewichtigen Empfehlungen gerne Gehör, die Neuerrichtete Universität in Melbourne, welche in London Sir John Herschel und Airy zu correspondirenden Mitgliedern hat, bietet, was man auch immer von dem Institute denken möge, die einzige Anknüpfung. Deßhalb frug ich an ob Se. Excellenz ein Ansuchen an die ebengenannten Herren [.? .] wie aus folgender Stelle hervorgeht: Bei Untersuchungen in der Richtung welche ich [.?.] Mangel an guten Instrumenten, die Grundbedingungen zu allen Beobachtungen. - Deßhalb stellte ich an Se. Excellenz die Bitte mir von Seiten der Königlichen Academie der Wissenschaften einige Instrumente zu erwirken, wodurch ich in den Stand gekommen sein würde unmittelbar zu beobachten und mir durch meine Resultate weiteren Eingang in Australien zu verschaffen und diese unbescheidene Bitte würde ich nicht gewagt haben, hätte nicht Herr Professor Dove mich selbst dazu aufgefordert und mir den Weg angezeigt, den ich darin befolgte. Nun erhalte ich keine Antwort auf mein Schreiben, das ich an ihn richtete. Ich überlasse es Ihnen, hochgeehrtester Herr Professor, über diese Handlungsweise sich Ihre Ansicht zu bilden. Wohl liegt in meiner Eingabe ein bestimmtes Unternehmen ausgedrückt: «Die Errichtung einer meteorologischen, magnetischen Station in Melbourne nebst einem Bureau für vergleichende Beobachtungen» und es muß diese Stelle Sr. Excellenz entgangen sein. Mit vagen Lebensplänen habe ich Se. Excellenz wahrhaftig nicht belästigen wollen - nur glaubte ich meine Triebfedern erkennen laßen zu müßen, weil heut zu Tage leider nur zu oft [.?.] ohne jedes ernstere Motiv, als weil sie sich in Europa unmöglich gemacht haben, in anderen Erdtheilen eine Wirksamkeit suchen.

\section{A.v. Humboldt und die Berliner Akademie gingen auf die Wünsche G. Neu- mayers zu einer konkreten Unterstützung seines Australienprojekts nicht}


ein, und Neumayer stand zuletzt mit leeren Händen da. Für den optimistischen und dynamischen jungen Rheinpfälzer war dies eine schmerzliche Enttäuschung, und er fühlte sich durch Humboldts Vorwurf, sentimental zu sein und vagen Lebensplänen nachzujagen, tief getroffen. Humboldt konnte hier Neumayers persönlichen Einsatz, die entsagungsvolle praktische Ausbildung eines Seemannes auf sich zu nehmen und nebenbei noch alle möglichen Kenntnisse über Schiffsführung, Schiffbau usw. sich anzueignen, nicht richtig einschätzen. Während dieser Jahre hätte sich Neumayer als reiner Naturforscher schon die ersten Lorbeeren verdienen können. Nun konnte er wissenschaftliche Leistungen noch nicht vorweisen, sondern nur um Vertrauen werben und seinen Idealismus für eine in seinen Augen patriotische Sache in die Waagschale werfen. Hier ist er im Einklang mit H.F.Maßmann, der aus der Jahnschen Turnbewegung kommt, in der Schweiz Johann Heinrich Pestalozzi besuchte und die praktisch-handwerkliche Ausbildung und Betätigung mit der geistig-wissenschaftlichen auf eine Stufe stellte ${ }^{20}$. Daß A.v. Humboldt sogar Empfehlungsschreiben Neumayer versagte, entsprach nicht seinem sonstigen Verhalten. Verständlicher mag dies werden, wenn man sich in die damalige Situation des «gequälten Helden der Wissenschaft» hineinversetzt, der trotz seiner Appelle, ihn mit Bitten um Stellungnahmen zu verschonen, von Manuskripten, Denkschriften usw. überflutet wurde ${ }^{21}$.

Obwohl Maßmann vor seiner Berliner Zeit in München bis 1842 wirkte, kam die Verbindung mit Georg Neumayer wahrscheinlich erst durch seine zwei Söhne zustande, die an der Hamburger Navigationsschule ihr Steuermannspatent erwarben ${ }^{22}$. Für Neumayer, in der Seemannschaft wie in der theoretischen Nautik gleichermaßen erfahren, stellte das von M. F. Albrecht und C. S. Vierow bearbeitete «Lehrbuch der Navigation und ihrer mathematischen Hülfs-Mittel» (1854 in Berlin erschienen) keinen Fortschritt gegenüber Ch. Rümkers Werk «Handbuch der Schiffahrts-Kunde mit einer Sammlung Seemanns-Tafeln» (5. Aufl. Hamburg 1850) dar - ein Werk, das S. Günther als das «erste, höheren Anforderungen genügende Compendium der wissenschaftlichen Nautik» bezeichnete ${ }^{23}$. Carl v. Littrow war als Leiter der Wiener Sternwarte auch an nautischen Forschungen interessiert. Neumayer hatte ihn im Sommer 1851 in Wien persönlich kennengelernt, als er nach Triest reiste, um dort bei der österreichischen Kriegsmarine eine geeignete Stellung zu finden ${ }^{24}$.

Während seines Australienaufenthaltes 1853 hatte sich Neumayer auch ein detailliertes Bild von den politischen Verhältnissen in der Kolonie 
Victoria gemacht, die in jenem Jahrzehnt eine parlamentarische Selbstregierung und die Zollfreiheit bekam. In Melbourne, dem kulturellen und wirtschaftlichen Mittelpunkt des Landes, wohnten verhältnismäßig viele Deutsche. Hier wirkte auch Ferdinand Jakob Heinrich Müller (1825-1896), 1871 vom Württembergischen König zum erblichen Freiherrn ernannt, als «Government Botanist». ${ }^{25}$

Es gibt aber möglicherweise noch einen anderen, ganz banalen Grund dafür, daß die Kommunikation zwischen G. Neumayer und A.v. Humboldt völlig in eine Sackgasse geriet. Der folgende Brief deutet darauf hin ${ }^{26}$.

\begin{abstract}
Sr. Wohlg.
Herrn Professor Maßmann

Dessauer Str. 22

Ich klage meine eigene Unordnung an, die bei 3500 jährlichen Briefen und Secretärmangel (weil ein [.?.] Secretär meine häusliche Ruhe stört und bald einen «Hofraths-Titel» fordern würde) einigermaßen zu entschuldigen ist. Liegt der Brief des braven Neumayer jetzt in Frankenthal bei Ihnen, theurer Freund? Er klagt über mein Stillschweigen. Ich will gern freundlich antworten, aber was? Er will, daß ich ihm Empfehlungen nach England gebe, damit ihm eine Lage in Australien geschaffen würde, von der er durch seine dortige Residenz und seine Navigationskenntniß nüzliche Berichte machen kann. Wie ist zu glauben, da nichts von einer speciellen Reise, von einem speciellen Unternehmen die Rede ist, daß von solch formloser Empfehlung an Faraday, Admiral Beaufort, Herschel und an die Geographische Gesellschaft irgend ein Eindruck [hinter]lassen wird. Ebenso gut konnte ich es $\mathrm{Hl}$ [Herschel?] im Sommer und der hiesigen Akademie vorschlagen. Wie sollte ich Engländern und Amerikanern, die täglich mit Australien in Verbindung sind, glauben machen, ein ihnen unbekannter Mensch besitze eigene Recepte. Geben Sie mir einen Rath, wie ich dem Mann nützlich sein kann. Auf eine sentimentale Correspondenz über vage Lebenspläne kann ich mich nicht einlassen.
\end{abstract}

Ihr

threuer

A.v. Humboldt

Montag 26.2.55

Hiernach ist fast mit Sicherheit anzunehmen, daß A.v. Humboldt die so ausführliche Neumayersche Denkschrift vom 6. Dezember 1854 überhaupt nicht zu Gesicht bekommen oder gelesen hat. Ob dies an Humboldts Überlastung und dem Alter lag oder ob das Schreiben bei Maßmann hängen geblieben ist, trotz seiner brieflichen Versicherung der Weitergabe, bleibe 
dahingestellt. Was aber mehr überraschen könnte, ist der durch den obigen Brief mögliche Aspekt, daß Humboldt es übersah, Neumayers Denkschrift vielleicht aus Zeitmangel nicht gelesen zu haben - oder weil sie gar unter irgendeinem Stapel unerledigter Briefe in Vergessenheit geraten war - und sein Urteil und seine Antwort nur auf dem Eindruck der lange zurückliegenden Unterredung mit Georg Neumayer und dem aus einer Notsituation heraus geschriebenen Briefe fußt. Kein Wunder also, daß Neumayer von dem völligen Übergehen seiner konkreten Pläne baß erstaunt und verletzt war.

Georg Neumayer hatte sich offenbar auch an die Berliner Akademie gewandt - auf Doves Rat hin - mit der Bitte, ihn mit Instrumenten auszurüsten. Vermutlich war die beigelegte Kopie der Denkschrift oder Eingabe vom 6. Dezember 1854 für H. W. Dove, den führenden Meteorologen in der preußischen Hauptstadt, oder für die Akademie gedacht. In dem von uns wiedergegebenen Brief an Maßmann beklagt sich Neumayer bitter darüber, daß er von Dove nicht einmal ein paar Zeilen als Antwort erhielt. Was können die Gründe für Doves Rückzug und Schweigen gewesen sein? Vielleicht kann uns die Lebensbeschreibung, die sein Sohn Alfred für die Allgemeine Deutsche Biographie verfaßte, hier weiterhelfen ${ }^{27}$. Alfred Dove betont ausdrücklich, daß seine Darstellung auf Originalpapieren und persönlichen Erinnerungen beruht. «Wenn H. W. Dove», so schreibt er, «die [spätere] Gründung der Norddeutschen Seewarte v. Freedens (1868), wie deren Ersetzung durch die großartige Reichsanstalt der Deutschen Seewarte 1876 mit vollster Sympathie und einflußreicher Förderung begleitet, ... machte [er so] zugleich eine ungerechtfertigte Unterschätzung wieder gut, mit der er früher das geschäftige Streben des Amerikaners Maury nach einer wegweisenden Meteorologie für das Meer betrachtet hatte».

In Berlin war damals die Zeit für Ideen und Pläne, wie sie Georg Neumayer hegte, noch nicht reif. Das konservative Preußen betrachtete den Besitz einer eigenen Hochseeflotte immer noch als Luxus. In dem marinebegeisterten Prinzen Adalbert (1811-1873) besaß es freilich schon einen angesehenen Fachmann für Seefragen. Er drängte darauf, daß Preußen seiner Führungsrolle im Zollverein, der sich bis an die Nordsee ausgedehnt hatte, auch auf der maritimen Seite gerecht werde. Aber der Prinz war vorerst nur ein Rufer in der Wüste. Die Hansestädte Hamburg und Bremen dagegen hatten ihren Seehandel, großenteils unter dem Schutze Großbritanniens, nach Lateinamerika, Afrika und Ostasien ausgedehnt, und es bestanden schon Linienreedereien via New York. 
Warum hat Georg Neumayer in seinen schriftlich niedergelegten Lebenserinnerungen, in Reden und Äußerungen die negative Resonanz des Berliner Kreises auf sein Australienprojekt zeitlebens verschwiegen? War es das eigene Gefühl, daß er als unbekannter Wissenschaftler damals zu viel verlangte? Oder wollte er sich nicht die Blöße geben, zurückgewiesen worden zu sein? Wir können hier nur Vermutungen äußern. Die aufbewahrten Briefentwürfe zeigen den Historikern, mit welch großen Schwierigkeiten Neumayer anfangs zu kämpfen hatte. Gleichzeitig stellen diese Dokumente den leisen Vorwurf an die Adresse damals einflußreicher Persönlichkeiten dar, daß man ihm nicht genügend Vertrauen entgegengebracht hatte.

Als Neumayer zu Beginn der siebziger Jahre in Berlin abermals auftauchte, hatte sich das Blatt zu seinen Gunsten gewendet. Mit seiner achtjährigen Tätigkeit an dem von ihm ins Leben gerufenen FlagstaffObservatorium in Melbourne und mit den Veröffentlichungen seiner Ergebnisse war er in der Wissenschaft ein bekannter Mann geworden ${ }^{28}$. In den «Mittheilungen aus Justus Perthes' Geographischer Anstalt» 1862 hatte August Petermann schon auf das «äußerst werthvolle Werk» Neumayers (Results of the magnetical, nautical and meteorological observations, Melbourne 1861) aufmerksam gemacht ${ }^{29}$. Bis 1869 folgten noch drei weitere Werke über Beobachtungen und Auswertung des Datenmaterials vom Flagstaff-Observatorium. 1867 hielt Neumayer zwei mit Beifall aufgenommene Vorträge in London über die Erforschung Zentralaustraliens, nämlich vor der Royal Society und der Royal Geographical Society, und legte eine Abhandlung vor, die in den Proceedings der R.S. dann gedruckt wurde ${ }^{30}$. Im Juli 1865 war G. Neumayer zur «Ersten Versammlung deutscher Meister und Freunde der Erdkunde» in Frankfurt am Main eingeladen worden und hatte dort seine Ideen zu einem meteorologisch-nautischen und hydrographischen Zentralinstitut vorgestellt und viel Sympathie hierfür gefunden. Daneben setzte er sich auf dieser Versammlung für eine Wiederbelebung der Antarktisforschung ein ${ }^{31}$. Wilhelm von Freeden, durch eine Erbschaft unabhängig geworden, setzte die Vorstellungen Neumayers mit der Gründung der Norddeutschen Seewarte (1868) in die Tat um, wenn auch diese zunächst nur ein subventioniertes Privatinstitut war. Gemeinsam entwarfen 1871 G. Neumayer und Wilhelm v. Freeden einen Organisationsplan, und auf der Versammlung der Deutschen Naturforscher und Ärzte in Rostock erläuterte Neumayer das Vorhaben ${ }^{32}$. Der vorher so zurückhaltende H.W. Dove war über die meteorologischen Arbeiten aus dem Flagstaff-Observatorium des Lobes voll ${ }^{33}$. Geschickt verstand es Neumayer, 
durch Vorträge in der «Gesellschaft für Erdkunde in Berlin» die Augen einflußreicher Männer auf sich zu lenken ${ }^{34}$. In den Astronomen Carl Bruhns (1830-1881) und Wilhelm Foerster (1832-1921), die beide A.v. Humboldt sehr geschätzt und gefördert hatte, fand Georg Neumayer Freunde, die ihm hilfreich zur Seite standen ${ }^{35}$. Die Reichsgründung 1871 veränderte die Machtverhältnisse in Mitteleuropa; aus der preußischen Kriegsmarine wurde die Kaiserliche Marine. Albrecht von Stosch (1818-1896), einer der fähigsten Offiziere der Armee, wurde zum Chef der Admiralität ernannt und der Aufbau der Flotte forciert. Stosch besaß das Vertrauen des Kronprinzen, des späteren Kaisers Friedrich III., und seiner Gemahlin, der englischen Köngistochter Viktoria, und galt lange Zeit als «Rivale» Bismarcks. Als Foerster von einer in der Admiralität neu eingerichteten Stelle eines Hydrographen hörte, sorgte er energisch bei A.v. Stosch dafür, daß Georg Neumayer den Posten erhielt; somit konnte auch Neumayer bei den «obersten leitenden Stellen» deutsche Kulturpolitik mitgestalten ${ }^{36}$.

Foerster und Neumayer gehörten zu dem Typ des Wissenschaftlers, der ein hohes organisatorisches Talent besitzt und Motor für die Verwirklichung neuer Konzepte sein kann. Beide verfolgten eine engere Verzahnung von Wissenschaft und Technik ${ }^{37}$. In Neumayer fand Foerster wertvolle Unterstützung für sein geplantes «Sonnenobservatorium», das später zum Astrophysikalischen Observatorium Potsdam erweitert wurde und das mit den Ideen und dem Wirken K.F.Zöllners und H. C. Vogels verknüpft ist ${ }^{38}$. Er schätzte die auf der Höhe der Zeit stehenden Fachkenntnisse Neumayers und dessen Weitblick. Denn in Berlin wollte in jenen Jahren noch manch einflußreicher Wissenschaftler nichts von den Zusammenhängen zwischen erdmagnetischer Tätigkeit, der elfjährigen Sonnenfleckenperiode, den Fakkelstürmen auf der Sonne und den Polarlichtern wissen.

In Berlin hatte Georg Neumayer 1872 die erste Stufe seiner Karriere erstiegen. 


\section{Quellen, Anmerkungen und Literatur}

1 Deutsches Museum in München Bibliothek, Urkunden- und Handschriftensammlung 6760/6761 Neumayer. Den Damen und Herren dieser Abteilung bin ich für ihre freundliche Hilfe zu großem Dank verpflichtet. Die Erlaubnis zur Veröffentlichung wurde mir erteilt. Schreibweise und Zeichensetzung der handschriftlichen Dokumente wurden übernommen (K.H. W.).

2 G.v. Neumayer, Auf zum Südpol, Berlin 1901, S.6, und z. B. L. Friederichsen, Georg von Neumayer, in: Mittheilungen der Geographischen Gesellschaft in Hamburg, Bd.24, Hamburg 1909, S.287-297, bes. S.288; und S.Günther, G.v. N. zum 80.Geburtstag, Festschrift der Pollichia, Bad Dürkheim 1906, S. 3.

3 P. Heidke, Neumayer als Deutscher und Gelehrter, in: Zum hundertsten Geburtstage von Georg von Neumayer, Beilage zum Juni-Heft 1926 der «Annalen der Hydrographie und Maritimen Meteorologie», S.1-17, bes. S. 6.

4 In der Zeitschrift: Auf weiter Fahrt. Selbsterlebnisse zur See und zu Lande. Begründet von Julius Lohmeyer, fortgeführt von Georg Wislicenus, Bd.5 (1907) S. 1-30.

5 Hans-Jochen Kretzer, Windrose und Südpol, Leben und Werk des großen Pfälzer Wissenschaftlers Georg von Neumayer, Pollichia-Sonderdruck, 2. ergänzte Aufl., Bad Dürkheim 1984, Autobiographisches Manuskript von G. N.: «Mein Prüfungsjahr», S. 75.

6 A. Lodewyckx, Die Deutschen in Australien, Stuttgart 1932, S. 49 u. 53. - C. E. Meinicke u. A.Petermann, Australien nach dem Stande der Geographischen Kenntniss in 1871, Erg. Heft No. 29 u. 30 zu Petermanns «Geographischen Mittheilungen», Gotha 1871.

7 K.H. Wiederkehr, Die hamburgische Seefahrt und die Einführung der meteorologischgeophysikalischen Navigation, in: Zeitschrift des Vereins für Hamburgische Geschichte Bd. 73 (1987), S. 1-26.

8 John Fletcher, Hrsg., Humboldt-Briefe in Australien, Melbourne 1983, S.14-15. Das Zitat und eine Kopie des Briefes erhielt ich von der Alexander-von-Humboldt-Forschungsstelle Berlin DDR, ebenfalls den Hinweis auf das Vorhandensein des Briefes von A.v. Humboldt an H.F.Maßmann, wofür ich (K.H.W.) herzlich danke. Herrn Dr. Dieter Hoffmann, Akademie der Wissenschaften der DDR, bin ich für hilfreiche Unterstützung ebenfalls dankbar (K. H. W.), ferner Prof. Dr. K. R. Biermann.

9 G. Neumayer, Auf zum Südpol, S. 6.

10 Hanno Beck, Gespräche Alexander von Humboldts, in: A.v. H., Werk und Weltgeltung, hrsg. von Heinrich Pfeiffer, München 1969, S.266.

11 Hanno Beck und Wolfgang-Hagen Hein, Alexander von Humboldts Rede 1829 in Sankt Petersburg, in: Die Dioskuren, Probleme in Leben und Werk der Brüder Humboldt, Mannheim 1986, S.199-222. - Peter Honigmann, Entstehung und Schicksal von Humboldts Magnetischem «Verein» (1829-1834) im Zusammenhang mit seiner Rußlandreise, in: Annals of Science 41 (1984) S. 57-86. - Karl Heinrich Wiederkehr, Über die Verleihung der Copley-Medaille an Gauß und die Mitarbeit Englands im Göttinger Magnetischen Verein, in: Gauß-Gesellschaft e.V. Göttingen, Mitteilungen Nr. 19 (1982) S. 15-35. - Ders., Über die Auffindung des nördlichen und südlichen Magnetpols der Erde, die Antarktisexpedition von James Clark Ross (1839-1843) und die Verbindung zu Göttingen, in: Gauß-Ges. Mitteil. Nr.20/21 (1983/84) S.7-38. - Ders., The «Göttinger 
Magnetische Verein» (Magnetic Association or Magnetic Union) and the Antarctic Expedition of James Clark Ross (1839-1843), in: Schröder, W. (Ed.) Historical events and people in geosciences Frankfurt/M. and Bern 1985 S. 73-79.

12 H.Ertel, Entwicklungsphasen der Geophysik. Berlin 1953. Sowie: Adrian Steleanu, Alexander von Humboldt und die Bedeutung seines wissenschaftlichen Werkes für die Hydrobiologie. In: Alexander von Humboldt, Gedenkschrift zur 100. Wiederkehr seines Todestages, Berlin 1959, 423-444 sowie daselbst H. Ertel: Zum Geleit, in: ebd., V. - Karl Schneider-Carius, Alexander von Humboldt in seinen Beziehungen zur Meteorologie und Klimatologie, in: A.v.H., Vorträge und Aufsätze anläßlich der 100. Wiederkehr seines Todestages am 6. Mai 1959, hrsg. von Johannes F. Gellert, Berlin DDR 1960. - Siegmund Günther, Geschichte der Erdkunde, Leipzig Wien 1904, S.315. - Eine bekanntere Humboldtsche Arbeit zur atmosphärischen Physik ist die über die nächtliche Verstärkung des Schalls (1820). Sie ist veröffentlicht in Kleinere Schriften, Bd.1: S.371-397. Eine quantitative Lösung der mit der Humboldtschen Entdeckung einhergehenden Problematik hat H. Ertel in seiner Schrift «Ein Problem der meteorologischen Akustik (Die tagesperiodische Variation der Schallintensität)» Sitz.-Ber. Dt.Akad. Wiss., Kl.f. Math., Phys. u. Technik 1955, Heft 2, gegeben.

13 Hanno Beck, Alexander von Humboldt als größter Geograph der Neuzeit, in: Die Dioskuren, Probleme in Leben und Werk der Brüder Humboldt, Mannheim 1986, S.126-182. - John Leighly, M. F. Maury, in: Geographers Bibliographical Studies, Vol. 1, ed. by T.W.Freeman, Marguerita Oughton and Philippe Pinchemel, Mansell 1977, S.59-63. Deutsche Übersetzung von C. Böttger, Die physische Geographie des Meeres, Leipzig 1856, neu herausgegeben von R.H.Francé, Leipzig 1911, S.15. - Arnold Schumacher, Gedenktage M.F. Maury und die Brüsseler Konferenz 1853, in: Deutsche Hydrographische Zeitschrift Bd. 6 (1953) S. 87-93.

14. M.F.Maury, Explanations and Sailing Directions to accompany the Wind and Current Charts, Washington 1852, S.3 u. 4. Unser Zitat ist nur der Anfang eines längeren Schreibens. Auch in einem von B. Silliman aufgezeichneten Gespräch wird berichtet, wie sehr A.v. Humboldt Maurys Werk anerkannte. Siehe hierzu Hanno Beck, Gespräche Alexander von Humboldts, Berlin (DDR) 1959, S. 304. - In Humboldts «Kosmos» sucht man vergeblich nach einem Hinweis auf Maurys maritimes Werk. A. Dove bemerkt aber in A.v. Humboldt, eine wissenschaftliche Biographie (hrsg. von Karl Bruhns), 3 Bde. Leipzig 1872, im 2. Bd. auf Seite 418, daß «Hydrologie und Meteorologie bedauerlicherweise nicht nach Maßgabe des Wissens von $1859 \mathrm{im}$ Kosmos abgehandelt wird».

15 Heinz Kautzleben, Die Förderung von Geodäsie und Geophysik durch Alexander von Humboldt und seine Wirkung bis in die Gegenwart. Abh. Akad. Wiss. (Berlin) N 2 (1986), 71-76. - Kurt-R. Biermann, A.v. Humboldts maritime Unternehmungen, in: Spektrum 1. Jg. (1970), hrsg. von der Deutschen Akademie der Wissenschaften zu Berlin DDR, H. 8 S. 34-35 ferner ders.: Mber dtsch Akad Wiss Berlin, Jg 13 (1971), S. 382-392.

16 A.v. Humboldt, Kosmos, Entwurf einer physischen Weltbeschreibung, 5 Bde., Stuttgart und Tübingen 1845-1862, bes. Bd. 5 S. 331.

17 Hanno Beck, Alexander von Humboldt als größter Geograph der Neuzeit (siehe 13).

18 Siehe Joh. Hanstein, Chr. G. Ehrenberg, Allgemeine Deutsche Biographie, 5. Bd. (1877) S.701-711, Neudruck Berlin 1968. - Rudolph Zaunick, Chr.G.E., Neue Deutsche Biographie Bd.4 (1959) S. 349 u. 350. 
19 G. Neumayer, Auf zum Südpol, S. 8 u. 11. Die Denkschrift an Godeffroy ist wiedergegeben in: K.H. Wiederkehr, Die hamburgische Seefahrt etc. (siehe 7).

20 Hans Ferdinand Maßmann (1797-1874) war einer der besten Schüler und Mitarbeiter des Turnvaters Jahn; sein Charakter und seine Zielsetzungen wurden wesentlich vom Gemeinschaftserlebnis der Jahnschen Sportveranstaltungen auf der Hasenheide beeinflußt. Von der nationalen Begeisterung der Befreiungskriege getragen, dichtete er Turnund Vaterlandslieder, neigte aber auch in jungen Jahren gelegentlich zum Radikalismus. In Bayern betraute man ihn 1827 mit der Einführung des Turnunterrichts. Er betrieb aber auch altdeutsche philologische Studien, suchte eifrig in Bibliotheken nach alten Texten und gab diese heraus. Die Münchner Universität ernannte ihn 1835 zum außerordentlichen Professor der deutschen Sprache und Literatur. 1842 holte man Maßmann zur Organisation des Turnunterrichts nach Berlin. Dieser Berufung war jedoch kein Glück beschieden. Eigensinnig verschloß er sich neuen methodischen Richtungen in der Leibeserziehung und schied aus seinem Amt bald aus. Hatte Maßmann schon früher Heinrich Heines ätzenden Spott ertragen müssen, so tauchten nun auch Gegner unter den Universitätsprofessoren auf. Der preußische König und A.v. Humboldt hielten aber zu ihm. Siehe hierzu Edmund Neuendorff, Geschichte der neueren deutschen Leibesübungen von Beginn des 19. Jahrh. bis zur Gegenwart, 4 Bde. Dresden 1930-1936, bes. 3. Bd. Hinweise werden Prof. Biermann verdankt.

21 Siehe hierzu Heinrich Sroka, Alexander von Humboldt Werke Briefe Selbstzeugnisse, Hamburg 1959, S.186 und Hermann Klencke, A.v.H. Ein biographisches Denkmal, Leipzig 1859, S. 118 K. R. Biermann, Alexander v. Humboldt, Leipzig 1987.

22 Maßmanns Sohn Friedrich besuchte 1860 den deutschen Turnverein in Melbourne als Steuermann auf dem Schiff «Sophie Friederike». Siehe hierzu Kl. C. Wildt, Daten zur Sportgeschichte, 4 Teile, Schorndorf bei Stuttgart 1970-1980, bes. T. 4 S. 152.

23 S.Günther, Rümker Karl Ludwig Christian, Allgemeine Deutsche Biographie 29. Bd., Neudruck der 1. Aufl. von 1889, Berlin 1970, S. 656 u. 657.

24. Carl Ludwig von Littrow (1811-1877), seit 1842 Direktor der Sternwarte und Professor der Astronomie an der Universität zu Wien, beschäftigte sich mit den Sonnendurchgängen von Venus und Merkur und mit Kometenbahnen. Siehe hierzu auch Johannes Georgi, Georg Neumayer (1826 bis 1909) und das 1.Internationale Polarjahr 1882/1883, in: Deutsche Hydrographische Zeitschrift, 17.Jg. (1964) S.249-270, bes. S.264, und H.J.Kretzer, S.72; Ferrari d'Occhieppo, K.L.v.Littrow, Österreichisches Biographisches Lexikon 1815-1950, 5. Bd., Wien 1972, S. 252 und 253. - Johann Gottlieb Friedrich von Bohnenberger (1765-1831), Professor der Mathematik und Astronomie an der Universität Tübingen und Adjunkt an der Sternwarte. Sein Buch «Anleitung zur geographischen Ortsbestimmung vorzüglich mittels des Spiegelsextanten» wurde von G.H.Jahn neu bearbeitet in Göttingen 1852 herausgegeben.

25 F.v. Müller stammt aus Rostock in Mecklenburg. Er erforschte die Australischen Alpen, das Murray-Tal u.a. und erwarb sich große Verdienste um die Erforschung der australischen Flora. Er gilt als der größte Botaniker dieses Erdteils. Den Melbourner Botanischen Garten machte er zu einem der bedeutendsten der Welt. Siehe hierzu A.Lodewyckx, Die Deutschen in Australien, Schriften des Deutschen Ausland-Instituts Stuttgart, Kulturhistorische Reihe Bd.32, Stuttgart 1932 S.208-212 und Deidre Morris, Mueller, Baron Sir F.J.H.v., Australian Dictionary of Biography, Vol.5 1851-1890 Melbourne 1974, 
S.306-308. - Die Universität in Melbourne wurde 1853 gegründet. George Biddell Airy (1801-1892) war Royal Astronomer in Greenwich und einer der berühmtesten Himmelsbeobachter seiner Zeit. Daneben widmete er sich auch der Erforschung des Erdmagnetismus und der Meteorologie. John Herschel (1792-1871), Sohn von Friedrich Wilhelm (oder William) Herschel, führte das astronomische Lebenswerk seines Vaters fort und machte in Physik und Chemie viele Entdeckungen. Zusammen mit E. Sabine organisierte er Ende der dreißiger und in den vierziger Jahren des 19. Jahrh. die Errichtung eines weltweiten Netzes meteorologischer und magnetischer Beobachtungsstationen.

26 Brief Nr.62 der Briefe A.v.Humboldt an H.F.Maßmann, in Besitze der Deutsehen Staatsbibliothek, DDR-1086 Berlin; eine Kopie wurde mir (K.H.W.) zugeschickt und die Editionsgenehmigung erteilt, wofür ich herzlich danke (K. H.W.).

27 A. Dove, H. W. Dove, Allg. Deutsche Biographie 48. Bd. 1904, S.51-69, bes. S. 66 u. 69. Richard Scherhag, H. W.D., Neue Deutsche Biographie Bd.4, Berlin 1959 S.92-93.

28 Siehe hierzu J.C.Poggendorff's Biographisch-Literarisches Handwörterbuch zur Geschichte der exacten Wissenschaften 3. Bd. Leipzig 1898 S. 964 u. 965, 4. Bd. 1904 S. 1068 u. 5. Bd. 1926 S. 900; ebenso H. J. Kretzer, Windrose und Südpol, S. 37-40.

29 Mittheil. aus J.Perthes' Geogr. Anst. über wichtige neue Erforschungen auf dem Gesammtgebiete der Geographie von Dr. A. Petermann, 1862 S. 199.

30 G. Neumayer, A scientific exploration of Central Australia, Proceedings of the Royal Society No. 102 (1868). - A.Petermann, Dr. Neumayer's Projekt zur wissenschaftlichen Erforschung Central-Australiens, in: Mittheil. aus Justus Perthes' Geogr. Anst., Gotha 1868, S. 442-453, bes. S. 443. - G.v. Neumayer, Meine Bestrebungen auf dem Gebiete der Geographie, in: Mitteilungen der Geographischen Gesellschaft in München, 1.Bd. München 1906, S.2-16, bes. S. 6 u. 7.

31 Den Vorsitz in Frankfurt führte Dr. Otto Volger, genannt Senckenberg, Gründer des «Freien Deutschen Hochstifts für Wissenschaften, Künste und allgemeine Bildung». Siehe hierzu Walter Horn, Die Anfänge der Deutschen Seewarte, in: Zeitschrift des Vereins für Hamburgische Geschichte Bd.58 (1972) S.45-81, bes. 51. - K. H. Wiederkehr, G.v. Neumayer (1826-1909), die Deutsche Seewarte in Hamburg und die Erforschung der Antarktis, in: Mitteilungsheft 1 Jg.4 (1985) des Arbeitskreises «Geschichte der Geophysik» in der Deutschen Geophys. Gesellschaft, S. 3-10.

32 G.N. u. W.v. Fr., Entwurf eines Organisationsplanes für das nautisch-meteorologische und hydrographische Institut, Die Deutsche Seewarte, auf der Basis der bereits in Hamburg bestehenden Norddeutschen Seewarte, Berlin 1871. - G. N., Vortrag auf der 44. Versammlung Deutscher Naturforscher und Ärzte, Rostock 1871, Der Weltverkehr zur See und die Geophysik in ihrer Wechselwirkung, Tageblatt.

33 P. Heidke, a. a. O., S.9.

34. G. Neumayer, Vortrag, Über ein neues Instrument für Messungen von Tiefsee-Temperaturen, in: Verhandlungen der Gesellschaft für Erdkunde zu Berlin 1873 Heft Nr.1 S. 20-22 und ders., Bericht über den Stand der Afrikanischen Expedition Heft Nr. 21873 S. 31-35.

35 C. Bruhns, Direktor der Leipziger Sternwarte, war Vorsitzender der Untersuchungskommission, die sich mit einer Reform der Norddeutschen Seewarte beschäftigte und die Einrichtung der Deutschen Seewarte als Reichsinstitut einleitete.

36 W. Foerster, Lebenserinnerungen und Lebenshoffnungen, Berlin 1911, S.131-133. Die 
Einflußnahme Foersters bei v. Stosch ist unseres Wissens bis jetzt noch von keinem Biographen Neumayers erwähnt worden.

37 H.Oleak, Wilhelm Foersters Verdienste um die Wissenschaftsorganisation. In: Sternzeiten, Band II. Berlin 1977, S.35-40. - W.Schröder, Wilhelm Foerster und die Entwicklung der solarterrestrischen Physik. Die Sterne 59 (1983), S. 348-352. - Dieter Hoffmann, Wilhelm Foerster - Astronom, Wissenschaftsorganisator und Begründer der «Urania», in: Au R 21 (1983) 1, S. 7-11.

38 Siehe hierzu Wilfried Schröder, Das Phänomen des Polarlichts, Erträge der Forschung Bd.218, Darmstadt 1984, S. 67 ff. - D. B. Herrmann, Zur Vorgeschichte des Astrophysikalischen Observatoriums Potsdam (1865-1874), in: Astronomische Nachrichten Bd.296, Berlin DDR 1975, S.245-259. Als Mitglied der Kommission zur Etablierung des Observatoriums schlug Foerster ursprünglich auch Georg Neumayer vor. - Für C. H. Vogel und K.F.Zöllner, siehe: Große Naturwissenschaftler, Handbuch, VDI-Verlag 1986, hrsg. von F. Krafft. K. H. Tiemann, Wilhelm Julius Foerster, in: Beitr. Astronomie und Physikgeschichte, Heft 61, Berlin DDR 1987, S.12-67.

\section{Summary}

\section{Georg von Neumayer's geophysical project in Australia} and Alexander von Humboldt

During his whole life, the German geophysicist and maritime explorer Georg von Neumayer (1826-1909) was fascinated by the antarctic continent and the nearby oceans. As a young man, he engaged as a sailor and mate to reach the oceans of the Southern Hemisphere. In 1854, he acquainted the aged Alexander von Humboldt (1769-1859) with his plan to install in Melbourne (Australia) an observatory for meteorology, navigation and earth magnetism. Neumayer brought forward scientific, practical and patriotic reasons for the installation of such an institution. Humboldt - otherwise a great promoter of young talents - did not support Neumayer, which was a considerable disappointment for him. He nevertheless attained his aim in 1857. The authors describe this episode, using as yet unpublished autographs from the German Museum in Munich as well as the A.v.Humboldt-Forschungsstelle and the Deutsche Staatsbibliothek in Berlin. 


\section{Résumé}

\section{Le projet géophysique de Georg von Neumayer en Australie et Alexander von Humboldt}

Le géophysicien et océanologue Georg von Neumayer (1826-1909) fut sa vie durant fasciné par l'Antarctique et les mers avoisinantes. Jeune homme et sans ressources, il se fit engager comme matelot pour pouvoir parcourir les mers de l'hémisphère Sud. En 1854, il présenta à Alexander von Humboldt (1769-1859), déjà très âgé, son projet de fonder à Melbourne une «station centrale pour la météorologie, la navigation et le magnétisme terrestre», en faisant valoir pour cela des raisons scientifiques, pratiques et patriotiques. Mais ce fut pour lui une grosse déception de ne pas rencontrer chez le grand savant l'appui qu'il avait espéré. Il trouva néanmoins le moyen de réaliser son projet (1857).

Les auteurs éclairent cet épisode en se fondant sur des documents autographes encore inédits du Deutsches Museum de Munich, de la Fondation A.v. Humboldt et de la Staatsbibliothek de Berlin.

PD Dr. Karl Heinrich Wiederkehr

Birkenau 24

D-2000 Hamburg 76

Dr. Wilfried Schröder

Hechelstr. 8

D-2820 Bremen-Roennebeck 